\title{
Balkanologie
}

Balkanologie Revue d'études pluridisciplinaires

Vol. IX, n' 1-2 | 2005

Volume IX Numéro 1-2

\section{Le meurtre du prêtre comme violence inaugurale (Bulgarie 1872, Macédoine 1900)}

Priest killing as first blood (Bulgaria 1872, Macedonia 1900)

Bernard Lory

\section{OpenEdition}

1 Journals

Édition électronique

URL : http://journals.openedition.org/balkanologie/572

DOI : $10.4000 /$ balkanologie. 572

ISSN : 1965-0582

Éditeur

Association française d'études sur les Balkans (Afebalk)

Édition imprimée

Date de publication : 1 décembre 2005

ISSN : 1279-7952

Référence électronique

Bernard Lory, "Le meurtre du prêtre comme violence inaugurale (Bulgarie 1872, Macédoine 1900) », Balkanologie [En ligne], Vol. IX, n 1-2 | 2005, mis en ligne le 13 janvier 2010, consulté le 17 décembre 2020. URL : http://journals.openedition.org/balkanologie/572 ; DOI : https://doi.org/10.4000/ balkanologie. 572

(c) Tous droits réservés 


\title{
LE MEURTRE DU PRÊTRE COMME VIOLENCE INAUGURALE (BULGARIE 1872, MACÉDOINE 1900]
}

\author{
Bernard Lory
}

La violence fait partie du projet révolutionnaire. Tous les programmes de libération nationale des peuples balkaniques au XIX ${ }^{\text {ème }}$ siècle envisagent de recourir aux armes afin de "libérer la patrie de l'oppression ottomane ". Cette violence programmatique est tournée contre un adversaire clairement défini : il s'agit du Turc, terme qui au XIX ${ }^{\text {ème }}$ siècle n'a pas encore pris sa signification ethno-nationale actuelle, mais qui désigne la population musulmane des Balkans, quelle que soit sa langue d'usage, dans la mesure où elle soutient le pouvoir impérial ottoman ${ }^{1}$. Les mouvements nationaux serbe, grec ou bulgare sont tous animés par la même idée motrice : le système ottoman des millets est inégalitaire et injuste, il faut donc le renverser ; le pouvoir doit passer des musulmans, qui sont minoritaires et " barbares " aux mains des chrétiens, majoritaires et engagés sur la voie du progrès.

La lutte que les révolutionnaires envisagent contre leurs adversaires désignés aura son prix et, dès le départ, des victimes sont à prévoir comme inévitables pour parvenir au but sacré, la liberté. Il faut être prêt à mourir pour la patrie, qui exige le sacrifice de ses meilleurs enfants. La rhétorique romantique du XIX ${ }^{e ̀ m e}$ siècle exalte puissamment la violence auto-sacrificielle.

Mais la violence révolutionnaire s'applique aussi à ses propres promoteurs. L'œuvre révolutionnaire est sacrée et la peine de mort est prévue pour les traitres et les insoumis. Les révolutionnaires prêtent des serments par lesquels

\footnotetext{
${ }^{1}$ La plupart des idéologues nationaux ont rédigé des documents annonçant qu'ils luttaient contre un pouvoir tyrannique et que les Turcs honnètes et exploités étaient invités à se joindre à leur lutte. Ces tex-

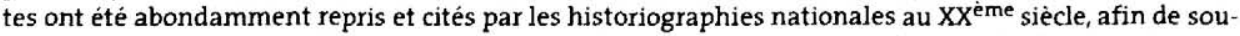
ligner la largeur d'esprit et le sens démocratiques de personnages qui sont devenus des héros nationaux. Si l'on examine l'action concrète des mouvements revolutionnaires, on constate que ce genre de propos n'avait aucune prise sur les mentalites des combattants.
} 
ils mettent leur propre tête en jeu. La révolution, dans les Balkans comme ailleurs, dévorera ses propres enfants².

Ces trois formes de violence (contre l'adversaire turc, sacrificielle, et contre les traitres) sont en quelque sorte statutaires et tout patriote qui s'engage dans la lutte doit être prêt à les assumer. Elles sont légitimées par la Cause sacrée. Très vite pourtant la violence révolutionnaire sort de ce cadre initial et nous la voyons frapper des gens qui ne sont ni des Turcs, ni des conspirateurs prêts à tous les sacrifices, mais des représentants de ce peuple-même, pour la liberté duquel la lutte est censée être menée.

L'objet de cet article est de cerner les mécanismes qui permettent ce glissement. À partir de quel moment des Bulgares tuent-ils d'autres Bulgares au nom de la cause nationale bulgare ? La même question sera posée au mouvement révolutionnaire macédonien, lequel imite, prolonge et pousse à l'extrême les tendances manifestées par le mouvement bulgare qui le précède d'un quart de siècle.

\section{PREMIER SANG VERSÉ EN BULGARIE}

La société ottomane dans laquelle vivent les Bulgares vers le milieu du $\mathrm{XIX}^{\mathrm{ème}}$ siècle est assez violente, même si on est loin des paroxysmes qu'a connus la fin du XVIII ${ }^{\text {ème }}$ siècle ${ }^{3}$. Au delà des outrances rhétoriques de la presse de l'émigration politique, la violence fait son apparition précocement dans la pratique révolutionnaire. La première phase du mouvement armé est celle des četa, de ces bandes armées infiltrées en Bulgarie ottomane depuis les territoires serbe ou valaque. Leur action implique de verser le sang. La četa de Panajot Hitov capture en 1867 , dès son arrivee sur le sol bulgare, un paysan turc, qu'elle exécutera de sang froid quelques jours plus tard. Vasil Levski, qui est le porte-drapeau (bajraktar) de la troupe, étrangle avec une corde le muhtar d'un village turc coupable d'exactions sur les chrétiens. Ces meurtres ont une vocation pédagogique visant à endurcir les combattants, bien plus qu'une fonction révolutionnaire quelconque. Les morts se succèderont ensuite, lors

\footnotetext{
${ }^{2}$ Deux ouvrages ont récemment été consacrés à la violence politique, celui de Markov (Georgi), Pokušenija, nasilie i politika v Bälgarija 1878-1947 (Attentats, violence et politique en Bulgarie 1878-1947), Sofia, 2003 et celui de Ačkovska (Violeta), Žeżov (Nikola), Predavstvata i atentatite vo makedonskata istorija (Les trahisons et les attentats dans l'histoire macédonienne), Skopje, 2004. Tous deux restent cependant dans le registre de la narration, voire de l'énumération, et ne cherchent pas à analyser les phénomènes qu'ils décrivent.

3 Nous avons abordé cette question dans : "Razsăżdenija vărhu istoričeskija mit "Pet veka ni klaha" " (Réflexions sur la mythe historique " Pendant cinq siècles on nous a massacrés "), Istoričesko bädešte, (1), 1997, pp. 92-98.
} 
des accrochages entre la četa et les forces de l'ordre qui la poursuivent. L'épisode le plus dramatique est celui où les četnik sont amenés à tuer euxmêmes leur camarade blessé Ivan Kapitanov4.

L'aspect sacrificiel est plus prononcé dans le cas de la četa de Hadži Dimităr, qui est anéantie par l'adversaire en 1868 , et dont certains membres se suicident par le poison, afin de ne pas être capturés vivants. Le sacrifice se retrouve dans la phase suivante du mouvement révolutionnaire, celle de l'édification d'un réseau de comités locaux clandestins. Un des épisodes les plus dramatiques en est le suicide d'Angel Kănčev, qui n'était âgé que de 20 ans, le 5 mars 1872 , sur les quais de Ruse, afin d'échapper à l'arrestation 5 .

En mai 1872, le Comité central révolutionnaire bulgare (CCRB) adopte de nouveaux statuts qui prévoient la peine de mort pour ceux qui tenteraient " de faire obstacle à la Cause, de quelque manière que ce soit " (chap. IX, art.5) ${ }^{6}$. Cela ouvre la porte à une violence bulgaro-bulgare, à laquelle aucune limitation précise n'est portée.

Le premier Bulgare assassiné au nom de la cause révolutionnaire l'a pourtant été quelques mois plus tôt, vers la fin de février 1872. Il s'agit de Stojan Penev, ancien typographe du journal Svoboda, publié en Roumanie par Lj. Karavelov, qui s'était vanté de pouvoir en dénoncer tous les collaborateurs aux autorités ottomanes. Mais c'est clairement à l'été de 1872 que le mouvement révolutionnaire bulgare opte pour le terrorisme, avec une stratégie d'extorsion de fonds, de menaces et d'exécution des récalcitrants. On connaît trois victimes : le čorbadži Vasil Kozlev de Ljaskovec, tué le 16 juillet, le diacre Paisij tué le 19 juillet à Orhanie7, et le domestique Stojčo Girginov poignardé par Levski à Loveč le 14 août.

Nous nous arrêterons tout particulièrement sur le deuxième cas, car il nous est décrit avec un grand luxe de détails, sous une forme légèrement romancée, par Stojan Zaimov. Cet activiste révolutionnaire (1853-1932) s'instaure comme mémorialiste du mouvement national ; il est moins célèbre que Zahari Stojanov, quoique leurs œuvres se ressemblent beaucoup. Tous deux ont eu à cœur, après 1878 , de faire connaître et de glorifier l'action du CCRB, afin de combattre l'idée démobilisatrice que " les Bulgares avaient reçu leur liberté des Russes ". En 1884-1888, il publie quatre volumes sous le titre de Minaloto

${ }^{4}$ McDermott (Mercia), The Apostle of Freedom. A Portrait of Vasil Levsky against a Background of Nineteenth Century Bulgaria, London, 1967, pp. 111-121.

${ }^{5}$ Dès 1881 , Zahari Stojanov consacre un article " aux héros qui se sont hardiment sacrifiés eux-mêmes pour leurs nobles principes et que tout patriote bulgare doit connaître, pour être fier de leur mémoire " (Stojanov (Zahari), " Imenata na bălgarskite văstanici koito sa posjagali sami na života si n (Les noms des insurgés bulgares qui ont attenté à leur propre vie) in Săčinenja, t. 2, Sofia, 1983, pp. 5-8.

${ }^{6}$ Undžiev (Ivan), Vasil Levski, biografija, Sofia, 1993 (1 ère éd. 1945), p. 549.

7 Le 21 selon Văzvăzova-Karateodorova (Kirila), Noneva (Zdravka), Tileva (Viktorija), Vasil Levski, dokumentalen letopis 1837-1873 (Vasil Levski, chronique documentaire), Sofia, 1987, p. 310, note 63. 
(Le passé), qu'il remaniera dans une seconde édition en 1898-1899. Le premier volume est quasiment entièrement consacré à l'affaire qui nous intéresse ici ${ }^{8}$. Qui est la victime ? Le diacre Paisij, natif de Trojan, est le vicaire général (namestnik) de l'évêque Ilarion de Loveč. Il s'intéresse de façon indiscrète aux activités du Comité révolutionnaire, répand de fausses rumeurs, incite ouvertement les villageois à payer leurs taxes ecclésiastiques, mais à ne rien donner aux représentants du Comité. Au physique, il est bel homme et Stojan Zaimov s'attarde à nous le décrire :

Son visage associe les traits de la beauté masculine : un nez byzantin, des yeux noirs, brillants comme des charbons ardents ; des sourcils noirs et épais qui se rejoignent ; une barbe et des moustaches noires et touffues, une bouche régulière, des dents blanches, claires et saines, à craquer des noix ; des joues pleines et rouges, comme des pommes de Kjustendil ; une taille moyenne, 25-27 ans d'âge. l'impression que produit ce jeune moine à qui le voit est celle que l'on éprouve quand on regarde un bouvillon de Pleven qui n'a pas encore connu le joug. Il est plaisant, agréable de regarder un taurillon, un jeune buffle ou un poulain bien nourris; de même il est plaisant de contempler un jeune et solide gaillard, un homme bien nourri. 9

Plus loin il nous est précisé qu'il a une belle voix lors de la liturgie ${ }^{10}$.

Dans ce diacre jeune, beau et mélodieux, le lecteur ne peut manquer de retrouver un véritable double de Vasil Levski, le héros absolu de l'histoire bulgare, qui fut connu comme le diacre Ignatij avant de devenir l'âme ardente du mouvement révolutionnaire. La première victime de la violence bulgaro-bulgare est donc le sosie de la figure emblématique du patriotisme, celui dont la mort est considérée comme une catastrophe pour l'histoire nationale ${ }^{11}$. La symétrie entre les deux figures n'est cependant jamais formulée de façon explicite. Il ne fait pourtant pas de doute à nos yeux qu'elle constitue un élément majeur de la dramaturgie mise en scène par St. Zaimov.

Or, Levski est le commanditaire du meurtre. Dans la première moitié de juillet 1872, il a ordonné par écrit l'exécution du diacre, soupçonné de trahison

${ }^{8}$ Nous utilisons l'édition de 1969 (Otečestven Front). Le meurtre du diacre Paisij est également documenté par le récit qu'en a fait l'assassin (lettre de D. Obšti datée du 22 juillet 1872, citée par Strašimirov (D. T.), Vasil Levski, život, dela, izvori, p. 363. note 329) et par Stojanov (P.I.), Gradăt Loveč, 1901 ; nous n'avons malheureusement pas pu effectuer la comparaison de ces versions. Á signaler que Stojan Zaimov fut probablement le premier Bulgare à travailler sur le thème aujourd'hui fort en vogue des lieux de mémoire ; il publia en effet en 1912 deux volumes intitulés Les lieux saints de la Bulgarie reconnaissante (Zaimov (Stojan), Svetite mesta na priznatelna Bălgarija, 1912).

9 Zaimov (Stojan), op.cit. p. 48.

${ }^{10}$ Ibid. pp. 148-149.

${ }^{11}$ Elle a fait l'objet d'un des poèmes les plus cẻlèbres de la littérature bulgare, dû à Hristo Botev. Une polèmique très violente sur la possible découverte de la sépulture de Levski a secoué les milieux journalistiques et scientifiques durant les années 1980 . La figure de Levski a été étudiée par de grands historiens contemporains comme Nikolaj Genčev et MarijaTodorova. 
envers la Cause $e^{12}$. Qui est l'exécutant ? Nous trouvons pour tenir ce rōle un des personnages les plus tristement célèbres de l'époque, Dimităr Obšti, celui-là même dont l'insubordination, les actions inconsidérées et la jactance finiront par causer la perte de Levski et par briser l'essor du mouvement révolutionnaire. Ce mouton noir de la geste patriotique est-il d'ailleurs vraiment bulgare ? Sa biographie, passablement compliquée, reste assez obscure : est-il originaire de Macédoine, du Kosovo ou même de Janina, comme l'affirmait la pancarte de son gibet (yafta) ? Son nom de famille (ou pseudonyme), Obšti, nie méme son identité (obšt = commun, général, non individualisé) comme un avatar néfaste d'Ulysse / Personne. Zaimov le décrit " de taille moyenne, le visage arrondi, des grands yeux bleus, un front haut et large légèrement ridé, de fines moustaches soyeuses, une musculature développée, une attitude gracieuse, vêtu avec tout l'attirail d'un dželep [fermier de l'impôt sur le bétail, commerçant aisé], un fusil à deux coups à l'épaule et tout un arsenal à la ceinture $n^{13}$.

Le cadre du drame est également décrit avec beaucoup de précision. Nous somme la veille de la Saint Elie de 1872, à Orhanie (aujourd'hui Botevgrad), bourgade récemment fondée par Midhat pacha, comme ètape sur la chaussée reliant Sofia et Pleven.

Les maisons de ce bourg sont comme accroupies des deux côtés de la chaussée qui le traverse de part en part. Parmi ces bâtiments accroupis le long de la chaussée, il y en a un qui se dresse tout droit alaturka et qui contemple fièrement, avec un sourire arrogant les bâtisses en bois accroupies autour de lui. Le nom de ce personnage arrogant est Šareni Han [1'Auberge Peinte]. Le han est la propriété de Stojan Brănčev, membre du conseil municipal d'Orhanie. On appelle ce bâtiment orgueilleux l'Auberge Peinte, non pas parce qu'il est badigeonné ou enluminé de quelques figures, de fleurs ou de décorations comme on en voit sur les fiers hôtels de Paris ou de Vienne, ba ba, pas du tout ; mais voici pourquoi : dans une des salles de cette auberge est dessiné un lion à sept têtes, cinq queues, cinq pattes et un seul œil au milieu du front. (...) À l'étage supérieur cinq à sept chambres pour les voyageurs sont réparties de façon irrégulière ; l'étage supérieur est pourvu d'un petit balcon (kiosque) en bois, qui fait saillie sur la façade méridionale. Du haut de ce balcon de bois on aperçoit très bien les sommets de la montagne, les crētes, les rivières, ruisseaux et gorges disposés au sud, sud-est et sud-ouest d'Orhanie (...) Plusieurs fois par an, la jeunesse d'Orhanie festoie sur ce petit kiosque de bois aux sons de la cornemuse bulgare, de la clarinette turque ou du crin-crin tsigane. ${ }^{14}$

${ }^{12}$ Văzuăzova-Karateodorova (Kirila), Noneva (Zdravka), Tileva (Viktorija), op. cit., p. 142.

I3 Zaimov (Stojan), op. cit., P. 52.

14 Ibid. p. 145 . 
Ce chaud soir d'été, un dîner est offert en ce lieu éminent au représentant de l'évêque par les notables d'Orhanie, qui sont membres du Comité révolutionnaire. Ils savent que le diacre Paisij a été condamné à mort et qu'il sera abattu au sortir de la féte. Le kaymakam d'Orhanie est également convié, en tant que représentant officiel du pouvoir. Le vin et la chaleur délient les langues et le kaymakam en vient à raconter le rêve qu'il a fait durant sa sieste de l'après-midi. Il a vu " Paisij, la tête et la barbe rasées, chaussé de babouches jaunes, qui [le] prie d'intervenir auprès de l' autorité compétente pour le faire admettre dans la vraie foi, c'est-à-dire qu'il veut se faire musulman. Le rêve du kaymakam était de ces rêves vivaces et nets, dont on garde longtemps l'impression en mémoire ${ }^{\mathbf{1 5}}$.

Or au même moment, ce mème après-midi, le diacre avait lui aussi fait un rêve troublant.

En rêve, le diacre était intronisé évêque. Tout était prêt pour l'intronisation, un seul élément manquait, mais il lui était impossible de savoir quoi. Cette chose inconnue empēchait de réaliser la cérémonie d'intronisation. L'image de la cérémonie se perdit tout à coup du cerveau somnolent et fut remplacée (comme dans une lanterne magique) par une nouvelle image qui n'avait rien à voir avec la première. Le diacre de l'évêque vit en rêve Paisij de Trojan, la tête et la barbe rasées, vêtu de la soutane d'un softa [étudiant en théologie] turc, un turban vert sur la tète, avec aux pieds, non ses bonnes chaussures habituelles, mais des pantoufles jaunes, et à la main, non une badine mais un long bäton de derviche. Le diacre se réveilla et comprit qu'il s'était vu lui-mème en rève. ${ }^{16}$

La concordance entre les deux rêves frappe tous les participants de la soirée, mais surtout ceux qui sont de la conspiration. Enfin, le diacre Paisij quitte la fête. Obšti en embuscade dans les fourrés est averti de son approche. La victime paraît, un premier coup fait long feu, un second touche le diacre au côté droit ; il meurt peu après, désignant le Comité comme responsable du meurtre, mais sans citer aucun nom.

Ce meurtre inaugural de la violence bulgaro-bulgare est donc annoncé par un rêve augural. Et même par deux rêves, simultanés et redondants, effectués par un chrétien et un musulman. Notre première réaction est bien sûr de scepticisme. St. Zaimov prend cependant soin de préciser en note : « De tous les participants au banquet, seul Petrov est décédé ; tous les autres sont encore en vie. Ils racontent avec un intérêt particulier la coïncidence des rêves. Merveilleuse est cette coïncidence entre les rêves et l'assassinat lui-même "17. Nous n'avons donc pas à faire à un procédé littéraire, assimilable aux nombreux dialogues

\footnotetext{
15 Ibid. p. 149

${ }^{16}$ Ibid. p. 149-150

${ }^{17}$ Ibid. p. 152
} 
rapportés ou inventés par l'auteur; le premier tome de Minaloto parait en 1884 , soit douze ans après les événements rapportés, ce qui réduit la liberté fictionnelle de Zaimov.

La symbolique des deux rêves est limpide : dans une société de millet, l'apostasie équivaut à une mort sociale, elle peut donc parfaitement symboliser la mort physique. Le converti (poturnak) est soumis à un ostracisme social qui le contraint à rompre avec son milieu d'origine, il est pratiquement considéré comme mort. Le passage d'un millet à l'autre est rendu manifeste par la barbe et la tête rasées et par le changement de costume, selon les codes vestimentaires en usage dans l'Empire ottoman. On note que le personnage conserve son statut d'homme de religion, malgré son changement de communauté, puisque de diacre, il devient softa ou derviche.

La redondance des deux rêves pose un problème. Le rêve de Paisij est assez explicite dans le contexte chrétien. Il rêve d'abord d'un changement de statut positif qui ne peut s'accomplir : son intronisation comme évêque (en sautant l'étape intermédiaire de hiéromoine). Puis il sort du registre chrétien et se voit en softa, ce qui constitue un changement de statut tout à fait négatif. Les deux scènes cumulées constituent un augure néfaste fort convaincant dans une optique chrétienne. C'est certainement ainsi que les conspirateurs du Comité révolutionnaire l'ont interprété.

En revanche pour le kaymakam (qui n'a pas de nom dans le récit de Zaimov) qui est musulman, un chrétien sollicitant son admission dans la vraie foi devrait a priori constituer un augure favorable. Le rêve du kaymakam ne fait sens que dans la mesure où il vient en appoint du rêve du diacre. Il fournit en quelque sorte la certification du caractère augural du rêve chrétien. Mais la démonstration ne devient bien sûr complète qu'à la fin du drame, après le banquet, lorsque Paisij est tué. Alors le caractère augural du rêve, que les conspirateurs chrétiens avaient identifié et que Paisij avait confusément pressenti, s'impose aussi pour le kaymakam musulman. Il devient clair, même aux yeux d'un musulman, que le passage d'un millet à l'autre, fût-ce une apostasie en faveur de l'islam, est bel et bien l'équivalent symbolique d'une mort. L'étrange histoire rapportée par Stojan Zaimov est une preuve quasi-scientifique, avec expérience (rêve $n^{\circ} 1$ ) et contre-expérience (rêve $n^{\circ} 2$ ) aboutissant au même résultat observable (la mort), que chrétiens et musulmans dans l'Empire ottoman partagent le même univers de références symboliques ${ }^{18}$.

Contrairement à ce que le lecteur pourrait croire, la sensationnelle histoire de l'assassinat du diacre Paisij n'est pas une des pages d'anthologie du récit historique bulgare. C'est au contraire un épisode peu connu et peut-être même

${ }^{18}$ Nous avons abordé cette question dans : " Dangers d'ici-bas, promesses d'au-delà. Essai d'anthropologie religieuse des Confins bosniaques au XVIIe siècle ", Ethnologia balkanica, 1 (1), 1997, pp. 173-177. 
occulté. Ce récit fondateur des pratiques violentes dans l'histoire nationale serait-il dérangeant ? Pour tenter de répondre à cette question, nous ferons un détour géographique et chronologique par la Macédoine.

\section{PREMIER SANG VERSÉ EN MACÉDOINE}

On date les débuts du mouvement révolutionnaire macédonien de l'automne de 1893 , avec la création de l'Organisation révolutionnaire intérieure macédono-andrinopolitaine (ORIMA). Ses fondateurs et ses premiers adhérents sont des enseignants du système scolaire bulgare financé par l'Exarchat. Ils ont lu et se sont passionnés pour les classiques de la littérature patriotique bulgare, et tout particulièrement les Notes sur les insurrections bulgares de Zahari Stojanov (trois tomes 1884-1892) et le roman Sous le joug d'Ivan Vazov (1889-189o) qui circulent sous le manteau dans l'Empire ottoman ${ }^{19}$. Le mouvement macédonien prend dès le départ pour référence le mouvement révolutionnaire bulgare, qui le précède d'un quart de siècle. Cette filiation est formulée explicitement dans les Statuts de l'Organisation ${ }^{20}$. Mais on la retrouve également, de façon implicite, dans son cheminement vers des formes violentes et fratricides.

L'évolution que nous avons retracée pour le CCRB se reproduit avec beaucoup d'analogies dans le contexte macédonien. Elle est toutefois compliquée par la rivalité entre deux organisations, l'ORIMA, qui agit de l'intérieur, tout en s'appuyant sur des bases arrières en Bulgarie, et le Comité suprême macédonien, qui agit à partir de la Bulgarie et qui est soumis aux intérêts politiques conjoncturels de la principauté bulgare. L'ORIMA consacre ses premières années à l'extension de son réseau conspiratif dans l'Empire ottoman, à l'établissement de canaux de communication avec la Bulgarie et à l'achat d'armes. L'initiative de la violence en Macédoine vient du Comité suprême, qui reprend à son compte la tactique des četa des années 1867-1868, en infiltrant de grosses bandes armées en territoire ottoman (opération contre Melnik en juillet 1895). Cette violence est tournée contre les Turcs, à savoir les petites garnisons ottomanes ou le village pomak de Dospat, mais aussi contre le patriarchisme et l'hellénisme, incarnés par l'évêque de Melnik. A partir d'avril 1896 (découverte de bombes à Bitola), mais surtout de novembre 1897 (Affaire de Vinica), les autorités ottomanes prennent conscience de l'ampleur du mouvement clandestin

19 Kosta Nikolov raconte dans ses mémoires que c'est Dame Gruev qui lui a fait lire Sous le joug (Nikolov (Kosta), Stranstvuvanijata na edin učitel (Les pérégrinations d'un instituteur) Sofia, 2001, p. 39).

${ }^{20}$ Siljanov (Hristo), Osvoboditelnite borbi na Makedonija (Les luttes de libération de la Macédoine), Sofia, 1983 (première édition 1933) t. 1, p. 39. 
et la répression policière s'abat sur les militants, les sympathisants, leurs proches ou des personnes complètement étrangères. La contre-violence d'État devance en quelque sorte la violence révolutionnaire, qui ne s'est pas encore véritablement manifestée, mais dont le trafic d'arme révèle clairement les intentions.

Vers 1898-1899, l'ORIMA inaugure une nouvelle tactique de četa : désormais elles ne doivent plus être injectées de l'extérieur pour des raids ponctuels, mais doivent se maintenir en permanence dans les zones montagneuses, afin de faire sentir leur présence auprès de la population. Ces četa permanentes, pour se donner une légitimité locale, procèdent à l'exécution de Turcs qui se livrent à des exactions sur la population chrétienne, en particulier des gardeschampê-tres, mais elles évitent de provoquer les forces de l'ordre. Les premiers affrontements sérieux ont lieu à Gavaljanci (région de Kukuš/Kilkis) le 20 mars 1898 et près de Valandovo, le 7 décembre 1899 . Ces combats ont des répercussions sur les comités locaux, qui subissent de nombreuses arrestations.

Le passage d'une violence extravertie, c'est-à-dire tournée contre l'adversaire programmatique turc, à une violence tournée contre l'adversaire intérieur, c'est-à-dire le traître, est plus difficile à cerner dans le cas macédonien que dans le cas bulgare. En effet, le camp des chrétiens était à peu près homogène dans la Bulgarie des années 1870. En Macédoine, il est au contraire très morcelé, puisque depuis une trentaine d'années déjà exarchistes (globalement pro-bulgares) et patriarchistes (globalement pro-grecs) s'affrontent sur la scène politique, mais on dépasse encore rarement le stade de coups de poings échangés. De nouveaux clivages apparaissent avec l'émergence du mouvement national aroumain et les progrès de la propagande nationale serbe dans les années 1890 . La rivalité entre l'ORIMA et le Comité suprême vient se surajouter à ces prises de position, dont il est bon de souligner qu'elles sont souvent très conjoncturelles et opportunistes, malgré des rhétoriques nationales essentialistes.

Les premiers assassinats politiques effectués par l'ORIMA sur des chrétiens concernent des serbomanes : Hadži Papa (alias Popović) poignardé, mais non tué dans les rues de Bitola le 25 octobre $1897^{21}$, Dimitrija Grdanov tué à Ohrid, le 5 août 1898 et Todor Popantov tué à Prilep le 6 janvier 1899. Ce sont bien des chrétiens slaves, des Macédoniens, qui sont exécutés par leurs concitoyens immédiats. Mais leur prise de position déclarée en faveur de la cause serbe fait d'eux des apostats de la cause nationale. Ayant abjuré leur communauté d'origine, ces victimes ne relèveraient donc pas encore vraiment du re-

21 " Thatsächlich folgen die Überfälle ähnlicher Art mit merkwürdiger Regelmässigkeit auf einander (Köprülü, Salonik, Perlepe, Monastir), so dass sie auf dieselbe Urheberschaft oder Leitung hinzuweisen scheinen ", H.H.St.A. Wien, P.A. XXXVIII, 389, Monastir, Kral, 27 octobre 1897 
gistre de la lutte fratricide. Sur le plan conspiratif pratique, ces serbomanes sont des traîtres, dont on craint qu'ils ne jouent le rôle de dénonciateurs auprès des autorités ottomanes.

S'il est malaisé de déterminer quand le " premier sang " est versé à l'intérieur du mouvement macédonien, il ne fait guère de doute que l'affaire qui a le plus de retentissement éclate durant l'été de 1900 à Bitola. On peut donc admettre que c'est elle qui inaugure l'ère des combats fratricides entre Macédoniens. À cette date, l'ORIMA connaît une crise financière, liée à son rapide développement. Les cotisations et les dons volontaires ne suffisent pas à assurer son fonctionnement. La nécessité de se procurer des armes est particulièrement pressante. Comme le CCRB trente ans auparavant, l'Organisation va recourir à l'extorsion de fonds par la menace.

En août 1900, un émissaire de l'ORIMA contacte le pope Stavre, vicaire général (ekzarhijski namestnik) de l'évêque Grigorij de Pélagonie, dont le siège est à Bitola ${ }^{22}$. Devant son refus de payer, la somme exigée est successivement augmentée de 15 à 30 livres turques, puis à 50 livres turques. Le pope aurait alors dénoncé le chantage auquel il était soumis aux autorités policières. Selon une autre version, ce serait le frère de sa bru, un Aroumain réputé espion turc du nom de Taki Čona, qui serait le délateur ${ }^{23}$. À son passage suivant, l'émissaire de l'ORIMA, un certain Trifun Ivanov du village d'Izbišta, près de Resen, est arrêté par la police ; il parvient néanmoins à faire disparaitre la quittance compromettante dont il était le porteur et n'est trouvé qu'en possession d'un poignard.

Le comité local décide alors de punir de mort le pope, considéré comme traître à la cause patriotique. Le $17 / 30$ août 1900 , il est abattu de plusieurs coups de revolver, sous les arcades de l'église exarchiste de la ville ; il meurt de ses blessures quelques jours plus tard. Les quatre membres du commando punitif et quinze autres militants seront jugés en mars 1901, à grand renfort de publicité, sous un chapiteau de cirque loue pour pouvoir accueillir un vaste public. L'assassin Trajko Petrev est condamné à mort, ses trois acolytes à la prison à vie. Dame Gruev, le responsable régional de l'ORIMA, est condamné à 10 ans de prison; il n'a pas pris part à l'attentat, mais la victime l'a désigné comme responsable moral ; il continuera d'ailleurs son activité subversive depuis la prison de Bitola, profitant de la négligence et de la corruption du système pénitentiaire ottoman.

Point de merveilleux, point de rêves prémonitoires dans ce sinistre règlement de comptes. Relevons cependant la signification du lieu du crime. $\grave{A}$

${ }^{22}$ La meilleure présentation de l'Affaire Pope Stavre est celle de Dimevski (Dimitar), Aferite vo Bitolskiot Vilaet 1895-1903 (Les " affaires " dans le vilayet de Bitola 1895-1903), Skopje, 1993, pp. 33-42, où l'auteur reprend et complète un précédent article : « Popstavrevata provala (1900 g.) i sudskiot proces vo Bitola (1901 g.) ", Istorija, 16 (1), 1980, pp. 131-140.

${ }^{23}$ Siljanov (Hristo), op. cit. p.111, note 1. L'exarque Josif, à Constantinople, rapporte dans son journal, sans trop y croire, que le délateur serait le fils du pope Stavre (Josif, Dnevnik, Sofia, 1992, p. 475, entrée du 24 octobre 1900). 
Bitola, ville où le meurtre politique se pratique couramment, c'est la rue qui est le théâtre de la violence. Les lieux les plus fréquemment cités sont le bazar (čaršija) et la grand rue (Hamidiye caddesi). Cela n'est guère surprenant pour une ville ottomane, qui connaît une relative ségrégation spatiale entre les communautés : il y a des quartiers d'habitation musulmans, chrétiens, juif, tzigane, mais la partie commerçante constitue l'espace commun, par conséquent aussi le lieu des affrontements. Le pope Stavre, au contraire, est assassiné à l'intérieur de l'enclos ecclésiastique de l'église de la Vierge (Sveta Bogorodica), espace que le Bulgar milleti s'est réservé, non sans difficulté, comme le sien propre dans le tissu urbain de Bitola ${ }^{24}$. Il tombe sous les balles de ses agresseurs sous le trem, la colonnade extérieure de l'église. Ceux-ci sont allés jusqu'à l'extrême limite de l'espace profane (pro fano : devant le temple) et ce meurtre n'a pas lieu à l'intérieur de l'espace sacré de l'église. Il reste donc en deçà du sacrilège mais, spatialement, s'en rapproche énormément ${ }^{25}$.

\section{CONTESTER L'ORDRE DES MILLET}

Le parallélisme entre les deux épisodes est tout à fait frappant. Deux organisations révolutionnaires récentes, arrivent au bout de quelques années de structuration interne, à une phase de crise. Crise financière d'une part, crise d'autorité d'autre part. Le recours à la violence est décidé. Le prétexte est lié à des questions d'argent, l'accusation portée est celle de trahison. Les deux affaires suscitent un vif émoi, parmi la population chrétienne, mais tout autant auprès de l'autorité ottomane.

Ce qui frappe le plus, c'est que les deux victimes exercent exactement les mêmes fonctions de vicaire général (namestnik), c'est-à-dire de $n^{\circ} 2$ dans leur diocèse. Le diacre Paisij est jeune (25-27 ans), il s'intéresse à la chose publique et envisage probablement une carrière épiscopale, ce qui, dans une société de millet, est le seul biais officiellement reconnu pour un chrétien d'exercer des fonctions représentatives dans l'Empire ottoman ${ }^{26}$. Il peut déployer ses talents

\footnotetext{
24 Lory (Bernard), " Un homme dans l'air du temps : Konstantin Michaykov (1807-1880), nationaliste et évergète à Bitola / Monastir n, in Anastassiadou-Dumont (Méropi), éd., Médecins et ingénieurs ottomans à l'âge des nationalismes, Paris, 2003.

25 On pourra objecter qu'un artisan aurait été abattu à la čaršija devant sa boutique, et que le pope Stavre trouve la mort sur son lieu de représentation sociale attendu.

${ }^{26}$ L'archimandrite russe Antonin Kapustin observe, lors de son voyage balkanique en 1865 : " Les diacres sont une rareté en Orient. Pour la plupart, les dignitaires ecclésiastiques de rang inférieur ne se voient qu'auprès des sièges épiscopaux, ils sont en général très jeunes, non mariés, avec de plus grandes capacités que les autres candidats, non pas pour la prêtrise, mais pour l'épiscopat. Ils forment l'escorte régulière des évêques orientaux, de même que de ceux d'ici [des Balkans], c'est pourquoi on les trouve auprès de leur métropolite à son siège épiscopal. " D'après la traduction macédonienne : Kapustin (Antonin), Makedonija vo delata na stranskite patopisci 1864-1874 (La Macédoine dans les œuvres des voyageurs étrangers 1864-1874), Skopje, 2000, p. 92.
} 
dans le diocèse de Loveč, car l'évêque titulaire Ilarion est retenu de façon durable à Constantinople pour les problèmes de mise sur pied de l'Exarchat bulgare.

Le pope Stavre est au contraire un homme d'âge mûr. Il était diacre vers 1863-1864, sous l'évêque Venediktos, qui l'ordonna prêtre vers 1868. Peu de temps après Stavre rompt avec son protecteur et s'engage dans le camp bulgare $^{27}$. Il est un des membres fondateurs de l'obština (cemât, conseil communautaire) bulgare à Bitola en 1868 , et il en assure la présidence à plusieurs reprises. On sait qu'en 1878 il cumule les fonctions de président de l'obština et d'ekzarhijski namestnik ${ }^{28}$. Aussi longtemps qu'il n'y a pas d'évẽque exarchiste à Bitola, c'est lui qui représente la communauté bulgare (slave non-patriarchiste) au plus haut niveau, dans la deuxième ville de Macédoine. À partir de décembre 1897, avec la nomination de Grigorij d'Ohrid au siège de Pélagonie (Bitola) le pope Stavre se retrouve $n^{\circ} 2$.

La création de l'Exarchat bulgare (Bulgar milleti) par firman impérial le 27 février/10 mars 1870 accorde au clergé orthodoxe bulgare une place prééminente au sein de la communauté, non seulement sur un plan politique et juridique, mais aussi sur le plan financier. La taxe ecclésiastique (vladičina) avait pendant des siècles principalement servi à alimenter les surenchères simoniaques du clergé phanariote, dont profitait, en dernière instance, le pouvoir ottoman. Dorénavant le clergé bulgare pouvait en disposer librement ; il en consacra une partie importante à son œuvre scolaire ${ }^{29}$. Cet argent communautaire, géré par le clergé, ne pouvait qu'attiser les convoitises des révolutionnaires, qui estimaient pouvoir mieux l'utiliser au nom de la cause nationale. Nous trouvons ce débat illustré de façon éloquente dans le roman d'Ivan Vazov Sous le joug, dans ce dialogue entre le révolutionnaire Ognjanov et le diacre Vincent :

- Demain il faut sans faute donner deux cents lires pour l'achat des fusils qui sont nécessaires à notre organisation. Si avant demain nous ne retirons pas ces fusils de $K$, un danger nous menace. Il faut trouver cet argent, et j'ai promis au comité de le lui procurer.

- Et que penses-tu faire, demanda le diacre

${ }^{27}$ Sapkarev (Kuzman), Za văzraždaneto na bälgarštinata v Makedonija (Sur la renaissance de la bulgarité en Macédoine), Sofia, 1984, pp. 230, 247.

${ }^{28}$ Kiril (Patriarh Bălgarski), Bälgarskata ekzarhija v Odrinsko i Makedonija sled osvoboditelnata vojna 1877-1878 (L'Exarchat bulgare en Thrace andrinopolitaine et en Macédoine après la guerre de libération de $1877-18787$, t. 1, livre 1, Sofia, 1969, pp. 555. 592.

29 Markova (Zina), Bălgarskata ekzarhija 1870-1879 (L'Exarchat bulgare 1870-1879), Sofia, 1989, pp. 166-200. 
- Il faut prendre cet argent au père Hiérothée.

- Mais comment ? Faut-il le lui demander ?

- Ce n'est pas ce que je veux dire, car de lui-même il ne le donnera jamais.

- Alors, que faire?

- Je te l'ai dit, il faut lui prendre cet argent.

- Autrement dit, il faut le lui voler ! s'écria le diacre.

- Oui, car il n'a nul besoin d'argent, alors que cet argent est nécessaire à la cause du peuple bulgare. Il faut le lui prendre, ou si tu préfères, le lui voler.

- Mais comment, Ognianov, un vol ?

- Oui, un vol, mais un vol sacré. "30

Ayant ainsi posé les termes de l'" expropriation révolutionnaire " le romancier recourt à un procédé un peu facile : le diacre Vincent est surpris, la main dans le sac, par son père spirituel ; mais ce dernier, derrière une apparence rugueuse, se révèle un franc patriote, qui finance des bourses d'études à de jeunes Bulgares à l'étranger et ne s'oppose pas cette " expropriation ". Le roman de Vazov contribue ainsi à minimiser l'antagonisme entre la voie évolutionniste et la voie révolutionnaire. De la confrontation brutale et sanglante que nous analysons pour 1872 et 1900 , il nous donne une vision édulcorée, où la notion vague de patriotisme permet de noyer les intérêts très divergents des différents promoteurs de la cause nationale. Il a pu contribuer à faire prendre à la légère la réalité des choses à ses lecteurs macédoniens. Il est fort probable que Boris Sarafov a cette version romanesque en tête, lorsqu'il se rend au Mont Athos pour y lever des fonds durant l'été de 1900, au moment même où la mort du pope Stavre est décidée. Faut-il préciser qu'il en revient bredouille ${ }^{31}$ ?

L'argent constitue-t-il d'ailleurs l'enjeu fondamental de la question qui nous intéresse ? On peut en douter. Le diacre Paisij dissuade, certes, les paysans de financer le comité révolutionnaire. Mais il adresse lui-même à Levski la modique somme de 5 mecidiye d'argent, quelques jours avant son exécution ${ }^{32}$. Quant à la somme de 15 livres turques, exigée dans un premier temps du pope Stavre, elle ne paraît pas exorbitante. Le comité révolutionnaire local fait une

\footnotetext{
${ }^{30}$ Vazov (Ivan), Sous le joug (traduction Roger Bernard et Nadia Christophorov), Paris, 1976, p. 295. Une lecture fine de ce chapitre montre que le meurtre du prêtre est envisagé de façon voilée par Ognjanov, porteur de l'idéal révolutionnaire.

" - Prends aussi ton couteau!

- Pour quoi faire?

- Tu peux en avoir besoin.

- Comment, je ne vais pas tuer quand même ! s'écria le diacre indigné.

- L'arme donne du courage. Veux-tu que je t'accompagne?

- Je ne veux pas de toi, bourreau ! dit-il avec une certaine animosité dans la voix n Ibidem p. 296.
}

${ }^{31}$ Siljanov (Hristo), op.cit., t.1, p. 89.

${ }^{32}$ Văzvăzova-Karateodorova (Kirila), Noneva (Zdravka), Tileva (Viktorija), op. cit., p. 141. 
évaluation qui nous semble réaliste de la somme qu'un prêtre de ville devrait pouvoir céder sans trop de difficultés. En même temps que Stavre, l'agent commercial bulgare à Bitola se voit réclamer 40 livres turques par le comité33. Tout juste un an après l'affaire qui nous intéresse, 10 livres est la somme que l'on extorque à un boulanger ou à un fourreur de Bitola 34 .

Non, l'argent est secondaire dans cette confrontation. L'accusation principale, dans la deux cas, est celle de trahison. Le diacre Paisij en est soupçonné, ou bien on l'en croit capable ; le pope Stavre est tenu pour responsable de l'arrestation d'un militant de l'organisation. Ni l'un, ni l'autre ne sont pourtant engagés dans le mouvement révolutionnaire, et ils n'ont prononcé aucun serment mettant en jeu leur vie. Ce n'est donc pas au nom de la "discipline révolutionnaire "qu'ils sont exécutés.

Mais quelle est alors cette "discipline patriotique supérieure" à laquelle ils se voient assignés ? Quelles sont les règles du jeu à l'intérieur de la communauté ? Qui définit les loyautés? Jusqu'en 1872, jusqu'en 1900 les cadres de la vie communautaire sont ceux de l'Exarchat bulgare, de ses règlements intérieurs et de la riche tradition juridique orthodoxe. Les organisations révolutionnaires, tout en prétendant agir pour la communauté, vont se placer en-dehors des règles communes. Le recours à la violence (considérée comme une violence légitime par les révolutionnaires) est une des marques de cette rupture. Les Statuts du CCRB de 1872, nous l'avons vu, légitimaient cette violence. Analogiquement, une réunion de 27 représentants de l'ORIMA, en août 1899 réclame des peines de mort contre deux évẽques exarchistes, Grigorij de Pélagonie et Gerasim de Strumica, ainsi que contre l'agent de l'Exarchat à Serres, Naumov. Le Comité central, toutefois, refusera de donner son approbation à ce projet 35 .

Dans les deux cas le meurtre du prêtre marque de façon claire la volonté des révolutionnaires de prendre le leadership au sein de la communauté, au détriment de ses représentants traditionnels. L'identification collective traditionnelle dans le cadre ottoman du millet, sous la houlette du clergé, est remise en question au profit d'une identification nationale lä̈que moderne. Cela se fait sans textes programmatiques et sans débats au sein de la communauté, par un acte dramatique et sanglant, dont la portée politique est immédiatement compréhensible pour tous. Le meurtre du prêtre, dans ces deux cas, est bien un meurtre fondateur, celui de l'État moderne. C'est un coup d'État.

\footnotetext{
33 La Bulgarie, principauté vassale de l'Empire ottoman, n'a pas le droit d'entretenir des consuls ; sans en avoir le titre, les agents commerciaux en remplissent les fonctions. Georgiev (Veličko), Trifonov (Stajko), Makedonija i Trakija v borba za svoboda (La Macédoine et la Thrace dans leur lutte pour la liberté) Sofia, 1995, pp. 36-37, note 27.
}

34 Cekalarov (Vasil), Dnevnik (Journal), Sofia, 2001, pp. 24-25.

35 Istorija na makedonskiot narod (Histoire du peuple macédonien), t. 2, Skopje 1969, p. 216. Un attentat contre Naumov échoue en avril 1900, Ibidem, p. 217 
Que le meurtre du diacre Paisij ait eu une valeur emblématique, et qu'il ait été conçu pour frapper l'opinion bulgare transparait dans une lettre adressée par Vasil Levski à Ljuben Karavelov, au moment où le CCRB se décide à passer à l'action violente : "Veličko efendi un de ces jours va tomber sur la tête. Nous retroussons nos manches ! et il me semble que l'on commencera par une tête noire $" 36$. Le passage n'est pas très facile à interpréter : Veličko est un notable de Šumen qui regimbe à financer les révolutionnaires, il est donc dans leur ligne de mire à Paisij, mais Levski veut-il dire que c'est parce que Paisij est membre du clergé qu'il doit être exécuté ? C'est peut-être forcer le sens d'un texte qui doit rester allusif, selon les règles de la clandestinité.

Deux exemples suffisent-ils à étayer notre interprétation du meurtre du prêtre ? Nos deux cas ont des précédents, mais nous sommes moins bien renseignés sur eux. On nous dit que Stefan Karadża, un des chefs de četa les plus respectés, voulut assassiner l'évêque grec de Tulcea vers $1864^{37}$. Nous savons aussi que le pope Tode (Todor) de Prilep fut agressè à la hache en février $1899^{38}$.

Une analyse plus fine des enjeux de pouvoir au sein de la communauté exarchiste de Bitola au moment de l'attentat contre le pope Stavre nous éclairera mieux sur les mécanismes du recours à la violence. Le firman instaurant l'Exarchat bulgare prevoyait que tout diocèse, dont les deux-tiers de la population orthodoxe en exprimait le désir pouvait rejoindre la nouvelle institution. Une consultation eut lieu dans le diocèse de Pélagonie, mais la crise d'Orient de $1875-1878$ vint interrompre le processus. Après la crise, le pouvoir ottoman ètait beaucoup moins bien disposé à l'égard de la cause bulgare, et il fit de l'attribution des berat épiscopaux un des principaux leviers de sa politique régionale. Les exarchistes de Pélagonie, quoique non reconnus sur le plan officiel, s'organisèrent sur le plan local, avec à leur tête un ekzarhijski namestnik, dont la position à l'égard des autorités restait assez ambiguë, puisque ces dernières considéraient l'évêque patriarchiste comme seul millet bașl légitime. Après la guerre gréco-turque de 1897 , la situation est modifiée. Le sultan accorde un berat pour le diocèse de Pélagonie et Grigorij, jusque là évêque exarchiste d'Ohrid, vient en occuper le siège. Il fait son entrée solennelle à Bitola le 3 janvier 189839 . C'est un homme réputé intelligent et énergique, mais aussi brusque et autoritaire, qui se rend vite impopulaire $4^{4}$. Il entre en conflit, non

\footnotetext{
${ }^{36}$ Undžiev (Ivan), op.cit., p. 551.
}

37 McDermott (Mercia), op.cit. p. 110.

${ }^{38}$ H.H.St.A. Wien, P.A. XXXXIII, 390 Monastir, Kral, 9 février 1899

39 H.H.St.A., Wien, P.A., XXXVIII, 389 , Kral, 10 janvier 1898.

$4^{\circ} \mathrm{Ibidem}, 23$ janvier 1898, 16 novembre 1898 . Voir aussi Trajanovskd (Aleksandar), "Otporot na bitolčani protiv postavuvanjeto na Grigorij za vladika na Pelagoniskata eparhija vo 1897 godina " (L'opposition des Bitoliens à la nomination de Grigorij comme évêque du diocèse de Pélagonie en 1897\}, Prilozi DNUB, \{z627), 1977 . 
seulement avec l'obština, mais surtout avec le comité local de l'ORIMA. Ses membres sont, pour l'essentiel des enseignants du système éducatif bulgare administré par l'Exarchat. Leur affectation professionnelle dans telle ou telle ville est un enjeu majeur pour le développement du réseau clandestin ; d'autre part, un grand nombre d'entre eux ne cache pas leur anticléricalisme, certains professent même leur athéisme.

Mais l'évêque n'est plus le seul représentant officiel de la communauté bulgare à Bitola. Depuis le mois d'avril 1897, une Agence commerciale bulgare s'est ouverte dans la ville, dont le responsable est le représentant de la politique de la principauté de Bulgarie. Sa légitimité est clairement d'ordre politique, et se réfère à une instance (le gouvernement de Sofia) qui est relativement lointaine, dans la perspective de Bitola. L'agent commercial bulgare de Bitola n'est pas impliqué dans la rivalité entre l'ORIMA et le Comité suprême macédonien (rivalité qui affecte peu la région de Bitola).

La communauté bulgare (slaves non-patriarchistes) est donc tiraillée entre trois autorités différentes : l'évêque, l'agent commercial et le comité révolutionnaire. Un premier incident se produit lors de la célébration de la Saint Cyrille et Méthode en 1899 : l'enseignant Cokov prononce un discours virulent contre l'évêque et se fait applaudir par une partie des enseignants et l'ensemble des élèves. Trois télégrammes identiques sont adressés à l'Exarque par Grigorij, l'agent commercial Mihajlov et le consul de Russie Rostkovski. Le licenciement de Cokov par l'Exarchat suscite une grève parmi les élèves, qui entraînera le renvoi de plusieurs d'entre eux et le licenciement des enseignants qui les ont appuyés ${ }^{41}$. C'est dans cette ambiance hautement échauffée que la peine de mort contre Grigorij est réclamée en août 1899 , lors de la réunion mentionnée plus haut. La question est jugée trop grave et transmise au Comité central. Goce Delčev se prononce contre la peine de mort ${ }^{42}$. Il est donc encore trop tôt pour procéder au meurtre du prêtre.

Mais l'intention du Comité révolutionnaire est connue. Et nous connaissons les réactions du clergé exarchiste 43 : le journal intime de l'exarque Josif mentionne dès septembre 1899 la menace d'assassinat contre Grigorij. Il demande à

${ }^{41}$ H.H.St.A., Wien, P.A., XXXVIII, 39o, Kral, 12 juin 1899

$4^{2}$ L'écrivain Anton Strašimirov rapporte, vingt ans plus tard, la crise morale que ce débat aurait causé à Goce Delčev. Vu la véritable hagiographie qui s'est développée autour du personnage, nous ne sommes pas enclin à accorder un grand crédit à ce témoignage (Strašimirov (Anton), Borbite v Makedonija i Odrinsko 1878-1912. Spomeni (Les luttes en Macédoine et en Thrace andrinopolitaine 1878-1912. Mémoires), Sofia, 1981, p. 699).

43 Nous ne connaissons malheureusement pas les réactions de l'Exarchat bulgare à l'assassinat du diacre Paisij. L'épisode, nous l'avons dit, n'a guère été traité dans l'historiographie bulgare. Il est totalement absent de l'étude de Săbev (Todor), " Bălgarskata pravoslavna cărkva i nacionalnoosvoboditelnoto dviženie " (L'église orthodoxe bulgare et le mouvement de libération nationale), in Aprilskoto văstanie $i$ bälgarskata pravoslavna cărkva, Sofia, Sinodalno izdanie, 1977, pp. 7-69. Il est également absent de l'étude de Markova (Zina), op.cit. 
Sofia " d'exercer leur influence sur le comité, afin qu'ils nous donnent leur parole qu'ils ne tueront pas Grigorij (...) Il est peu probable qu'ils tuent Grigorij, mais (...) ils le détestent et ils feront des scandales jusqu'à ce qu'ils s'assurent qu'il ne leur mettra pas de bảtons dans les roues. D'une façon générale, la situation n'est pas encourageante " 44 . Dans son bilan de l'année 1899,1 'Exarque analyse ainsi le rapport de forces : « Qu'on le tue, je n'y crois pas, mais qu'on lance des rumeurs pour l'effrayer, pour le soumettre à leur propagande et pour qu'il ne leur fasse pas opposition, ça j'y crois ". Il nous apprend également que l'évêque Avksentij de Veles vit dans la terreur d'être assassiné, que Grigorij, même à Constantinople, est constamment armé d'un revolver et n'ose pas sortir dans la rue 45 .

Avant même le meurtre du pope Stavre, la menace de violence oblige donc déjà la structure ecclésiastique de l'Exarchat à composer ${ }^{46}$. Alors pourquoi tuer Stavre ? Nous avons vu que dans leur campagne de levée de fonds les militants de l'ORIMA s'étaient adressés simultanément au pope et à l'agent commercial bulgare. Ce dernier repousse avec indignation la tentative de chantage 47 . Nous formulons donc l'hypothèse que le meurtre du pope Stavre ne doit pas servir uniquement à terroriser les milieux de l'Exarchat, mais que c'est aussi un signal indirect de mise en garde adressé à Sofia.

L'affaire pope Stavre ne s'arrête pas aux coups de feu devant l'église de Bitola. L'Exarque réagit en adressant des courriers répétés au gouvernement de Sofia et au prince Ferdinand, mais sans grand résultat. En effet, le clivage entre Comité suprème et ORIMA laisse peu de moyens de pression à Sofia sur cette dernière. En revanche les protestations de l'Exarque auprès de l'ambassade de Russie entraineront une note sévère de Saint Pétersbourg à Sofia.

Nous avons formulé l'hypothèse que le meurtre du prêtre est le crime fondateur du pouvoir laïc du futur État national sur le système ancien des millet. Il nous éclaire aussi sur la délicate question des rapports entre l'Église et l'État dans le contexte de l'orthodoxie balkanique. Il est clair que nous sommes loin du schema irénique que les théologiens s'efforcent de nous donner d'une coresponsabilité du basileus et du patriarche pour la défense de la foi. Si ce schéma a pu être valable à l'époque byzantine, il ne nous aide en rien à comprendre la situation dans les Balkans à l'époque des nationalismes. Le mécanisme que nous avons tenté de dégager montre au contraire que, dès les premiers balbutiements d'un pouvoir laïc, c'est la violence et la terreur qui ont été exercées sur le clergé, afin que la subordination du pouvoir religieux devienne immédiatement claire pour l'ensemble de la population.

44 Josif, op.cit. p. 455 .

45 Ibidem, p. 462. Le pope Śkutov de Lerin/Florina craint également pour sa vie, Ibidem p. 476.

$4^{6}$ Istorija na makedonskiot narod, t. 2, p. 218.

47 Georgiev (Veličko), Trifonov (Stajko), op.cit. 